\title{
Intraoperative localization in minimally invasive surgery for small pulmonary nodules: a retrospective study
}

\author{
Binjie Zhang, Yongkui Zhang, Hanbo Le, Wujun Li, Cheng Chen, Renxiu Fang, Xinfu Pan \\ Department of Thoracic Surgery, Zhoushan Hospital, Zhejiang Province, Zhoushan, China \\ Contributions: (I) Conception and design: B Zhang; (II) Administrative support: Y Zhang; (III) Provision of study materials or patients: H Le; (IV) \\ Collection and assembly of data: W Li; (V) Data analysis and interpretation: C Chen; (VI) Manuscript writing: All authors; (VII) Final approval of \\ manuscript: All authors. \\ Correspondence to: Xinfu Pan; Hanbo Le. Department of Thoracic Surgery, Zhoushan Hospital, Zhejiang Province, Zhoushan 316000 , China. \\ Email: mastermed@163.com; 324596708@qq.com.
}

\begin{abstract}
Background: Small pulmonary nodules are increasingly detected at an earlier stage and need to be removed via video-assisted thoracoscopic surgery (VATS). However, small pulmonary nodules are often difficult to locate during VATS and are typically nonvisible and nonpalpable on the lung surface. A variety of localization techniques have been developed. Here, we explored the application of an intraoperative body surface localization (IOBSL) and/or anatomical landmark localization (ALL) in minimally invasive surgery for small pulmonary nodules.

Methods: A total of 174 patients with small pulmonary nodules were divided into 3 groups: an IOBSL group, an ALL group, and an IOBSL+ALL group. VATS partial pneumonectomy was performed after the nodule localization, and the need for pulmonary segmentectomy/lobectomy and lymph node dissection was assessed according to the results of intraoperative rapid frozen section diagnosis. The duration, accuracy, and complications of each localization method were recorded and analyzed.
\end{abstract}

Results: ALL had shorter distance to the nodules $(\mathrm{P}=0.0282)$ but longer localization duration $(\mathrm{P}<0.05)$ than did IOBSL. The IOBSL+ALL group had higher localization accuracy than did the other 2 groups $(\mathrm{P}=0.0003)$ but with longer localization duration $(\mathrm{P}<0.001)$. No intraoperative complications were noted.

Conclusions: The intraoperative technique has high localization accuracy and a low complication rate. It can be applied in VATS for pulmonary nodules, depending on the specific locations of the nodules.

Keywords: Video-assisted thoracoscopic surgery (VATS); pulmonary nodule; intraoperative; localization

Submitted Mar 31, 2021. Accepted for publication Jul 19, 2021.

doi: $10.21037 /$ tcr-21-1059

View this article at: https://dx.doi.org/10.21037/tcr-21-1059

\section{Introduction}

With the rapid advancement of medical imaging technology, especially that of three-dimensional (3D) reconstruction algorithms for computed tomography (CT) images, the early detection of small pulmonary nodules has increasingly become a possibility. Many small pulmonary nodules are early lung cancer and require video-assisted thoracoscopic (VATS) resection. However, small pulmonary nodules are often difficult to locate during thoracoscopy, are undetectable on the lung surface, and challenging to palpate. Several pulmonary nodule localization techniques have been developed, including preoperative CT-guided hookwire metal localization, CT-guided puncture followed by injection of a marking substance (e.g., methylene blue), electromagnetic navigation bronchoscopy, and fluorescent staining. However, these localization methods are limited by their invasiveness; in addition, they are performed before surgery, and thus a time lag exists before the surgery is finally performed. Therefore, noninvasive or microinvasive intraoperative localization techniques are required to overcome the shortcomings of preoperative localization. In recent years, the minimally invasive intraoperative body 
surface localization (IOBSL) and/or anatomical landmark localization (ALL) have been applied in our center during VATS pulmonary sublobectomy for small pulmonary nodules, with satisfactory effectiveness. In this study, we explored the efficiency of IOBSL and/or ALL in minimally invasive surgery for small pulmonary nodules. We present the following article in accordance with the STROBE reporting checklist (available at https://dx.doi.org/10.21037/ tcr-21-1059).

\section{Methods}

\section{Subjects}

The clinical and imaging data of 174 patients with small pulmonary nodules who were treated in our center from January 2017 to June 2020 were retrospectively analyzed. Among them there were 69 males and 105 females, aged $27-82$ years, with an average age of $59.20 \pm 11.93$ years. A total of 221 nodules were identified, of which 181 were single, 27 were double, and 3 were multiple. The nodules were located in the upper lobe of the right lung in 63 cases, in the middle lobe of the right lung in 14 cases, in the lower lobe of the right lung in 30 cases, in the upper lobe of the left lung in 57 cases, and in the lower lobe of the left lung in 29 cases. The distance of the nodules to the pleura was $\leq 0.5 \mathrm{~cm}$ in 78 cases, $0.6-1 \mathrm{~cm}$ in 37 cases, $1.1-2 \mathrm{~cm}$ in 66 cases, and $>2 \mathrm{~cm}$ in 7 cases. The pathological findings included transient (TRA) lesions in 10 cases, atypical adenomatous hyperplasia (AAH) in 3 cases, adenocarcinoma in situ (AIS) in 42 cases, minimally invasive adenocarcinoma (MIA) in 77 cases, and invasive adenocarcinoma (IAC) in 79 cases. The localization duration and distance to the nodules among the 3 groups were recorded and analyzed. The study was approved by the ethics committee of Zhoushan Hospital with an approval number of [2018] ZHECA [163]. All procedures performed in this study involving human participants were in accordance with the Declaration of Helsinki (as revised in 2013). Individual consent for this retrospective analysis was waived.

\section{Localization techniques}

\section{IOBSL}

Before surgery, the CT images of the patients were read in the imaging system. The localization sites of the lung nodules on CT and chest wall surface were used as the localization points. The sites for needling or electrocoagulation were selected according to the principle of "near verticality". The distance between the localization point on the body surface of the chest wall and the anterior (posterior) midline was accurately measured. Additionally, the intercostal spaces corresponding to the selected localization points on the body surface of the chest wall were measured (Figure 1).

During operation, after successful general anesthesia, the patient was placed in a $90^{\circ}$ lateral position on the healthy side and marked according to the preoperatively selected body surface localization points. Disinfection and draping were routinely performed in the surgical field. An observation port was first created for inserting the thoracoscope. For sites where the chest wall was thin or not covered by the scapula, endoscopic observation was performed before the trocar needle was applied, which was inserted through the localization point and stopped $1 \mathrm{~cm}$ into the chest cavity. After the thoracoscope was withdrawn, the anesthesiologist was asked to inflate the lungs and hold it. The metal inner core on the tail of the trocar needle was cauterized with electrocoagulation so that the metal needle tip stabbed into the lung tissue marked the lung surface. For pulmonary nodules located in thicker parts of the chest wall or in obstructed sites, such as the scapula and ribs, a trace of electrocoagulation cautery was first left on the corresponding point at the pleura of the inner chest wall layer with the help of the localization point on the body surface of the chest wall; subsequently, the electrocoagulation hook was placed at the point, and the anesthesiologist was again asked to maintain the lung inflation before a cautery point was left on the lung surface with electrocoagulation cautery. Then, the thoracoscope was inserted to locate the cautery point, which was the localization point of the pulmonary nodule. Finally, the lesion was removed according to the surgical plan (Figure 2).

\section{ALL}

$3 \mathrm{D}$ reconstruction (mainly including transverse, coronal, and sagittal positions) of CT scan data were used to determine the $3 \mathrm{D}$ positions of small pulmonary nodules in pulmonary lobes and segments from different directions and dimensions (e.g., anterior-posterior, left-right, and updown) and to measure the distance between the pulmonary nodules and the anatomical markers. After the collapse of lungs during the surgery, many markings (such as the transverse and longitudinal axes, which are similar to the longitudinal and latitudinal lines on a globe) were visible on the lung surface. The longitudinal axis lines refer to the lines connecting the lung apices and the lung bases, 

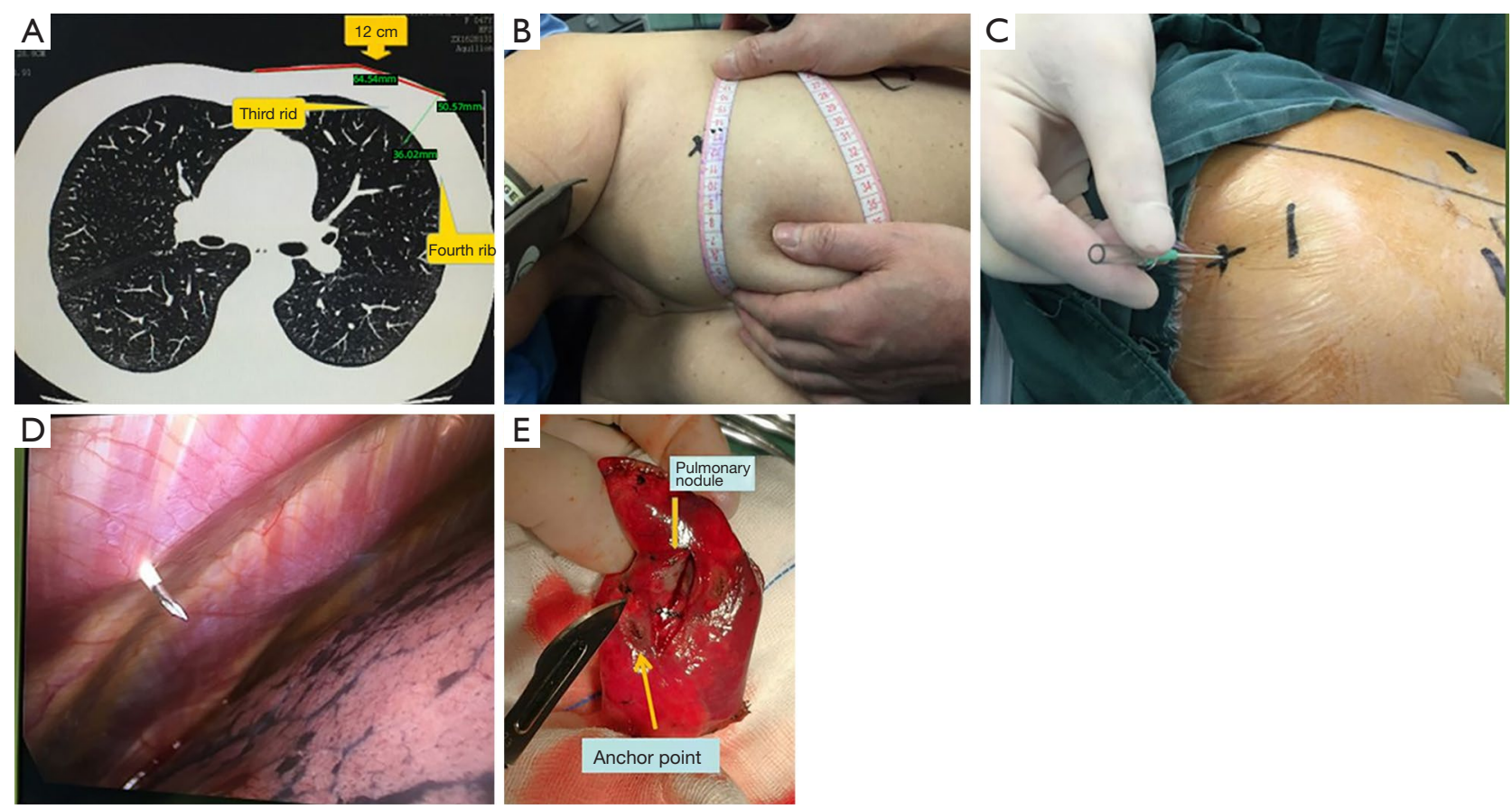

Figure 1 The operation process and illustration of the intraoperative body surface localization. (A) Preoperative measurement of the intercostal spaces where the pulmonary nodules were located on CT (the corresponding location and distance). (B) After anesthesia, the body surface locations corresponding to the pulmonary nodules were measured based on the localization points on CT. (C,D) The trocar needle was inserted via the preoperatively selected body surface localization points, and electrocoagulation was performed after the lungs were inflated to leave electrocoagulation marks on the lung surface. (E) The location where the nodule specimen was harvested and the distance to the localization point. CT, computed tomography.
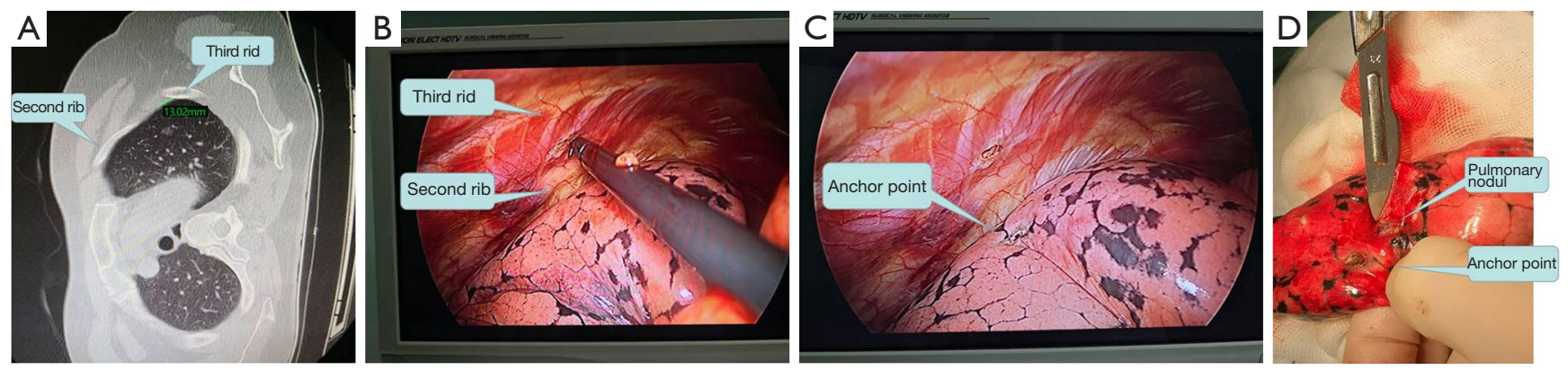

Figure 2 The operation process and illustration of the anatomical landmark localization. (A) Preoperative reading of the intercostal spaces corresponding to the pulmonary nodules on CT. (B) Intraoperative selection of the localization points in the thoracic cavity according to the preoperatively identified intercostal spaces corresponding to the small pulmonary nodules. (C) Localization point left on the surface of the lung by electrocoagulation following lung inflation. (D) The location where the nodule specimen was harvested and the distance to the localization point. CT, computed tomography. 


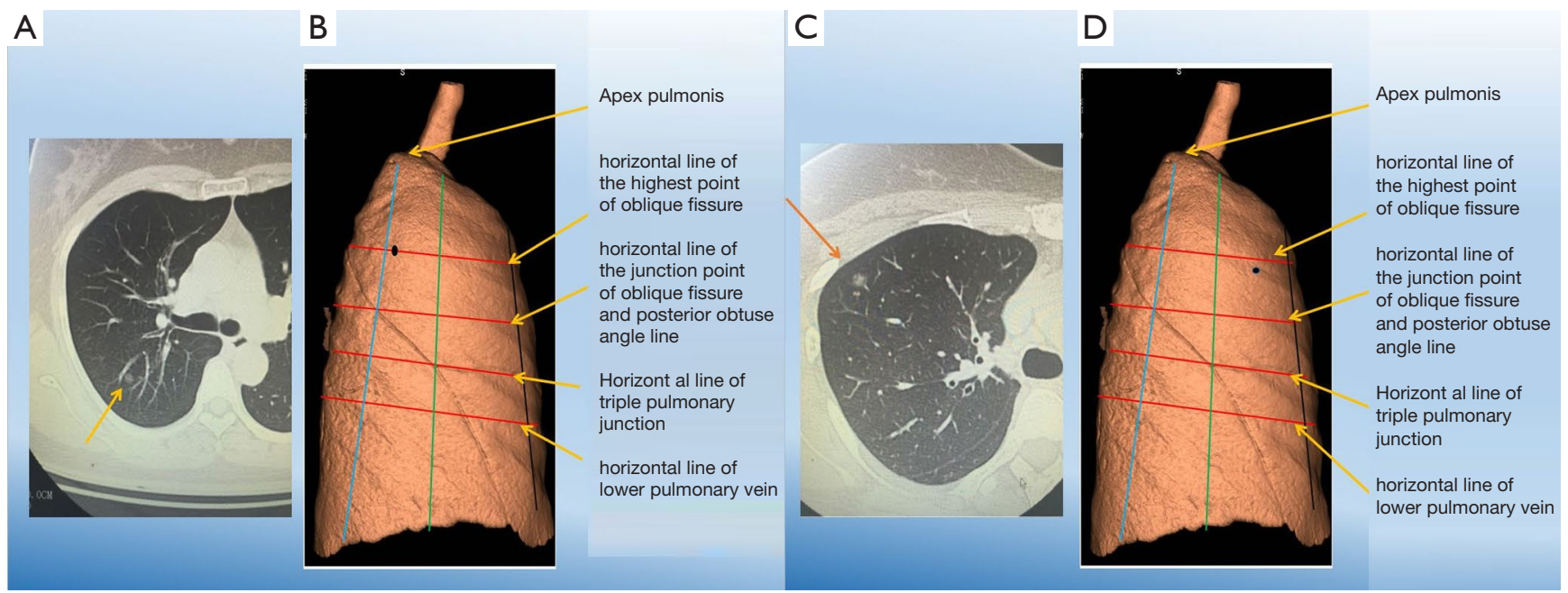

Figure 3 The demonstration of the latitude-longitude localization. (A) Preoperative reading of the axis lines corresponding to the pulmonary nodules on CT. (B) Graph-based simulated positions corresponding to the nodules. (C) Preoperative reading of the axis lines corresponding to the pulmonary nodules on CT. (D) Graph-based simulated positions corresponding to the nodules. The orange arrow in $(\mathrm{A}, \mathrm{C})$ indicates the pulmonary nodules on CT. CT, computed tomography.

primarily the acute angle line, the anterior obtuse angle line, the vertical line where the two lungs meet, the posterior obtuse angle line, the posterior internal obtuse angle line, and the paraspinal line. The horizontal axis lines refer to the horizontal lines on the surface of the lungs, primarily the horizontal line of the azygous vein, the horizontal line of the ramus, the horizontal line of the highest part of the oblique fissure, the horizontal line of the junction between the oblique fissure and the posterior obtuse angle line, the horizontal line of the junction point among the three lungs (right lung), the horizontal lines of the upper, (middle) and lower pulmonary veins, and the bases of the lungs. These lines were calculated proportionately and marked on the surface of the diseased lung. A relevant article on this procedure has been published by our team (1) (Figure 3).

\section{VATS}

The surgery was performed through a single operating port, although the incision sites differed according to the locations of the pulmonary nodules. For the upper pulmonary nodules, typically the observation port was created in the sixth intercostal space in the midaxillary line, whereas the observation port was placed in the third intercostal space in the anterior axillary line. For the lower pulmonary nodules, the observation port was created in the seventh intercostal space in the midaxillary line, whereas the observation port was placed in the fourth intercostal space in the anterior axillary line. The lesions were routinely removed based on the results of intraoperative localization.

\section{Statistical analysis}

The data were collected and analyzed with SPSS 19.0 software. The differences among three groups were analyzed with one-way ANOVA assay followed by Turkey's multiple comparison test. $\mathrm{P}<0.05$ were considered statistically significant.

\section{Results}

\section{Comparisons among 3 intraoperative localization methods}

The localization points were significantly closer to the nodules in the ALL group than in the IOBSL group $(\mathrm{P}<0.05$; Table 1), whereas the duration of localization was significantly longer in the ALL group than in the IOBSL group $(\mathrm{P}<0.01$; Table 1$)$. Compared with the IOBSL group and ALL group, the IOBSL+ALL group showed a significantly shorter distance to the nodules $(\mathrm{P}<0.01$; Table 1$)$ but a longer duration of localization $(\mathrm{P}<0.001$; Table 1).

\section{Discussion}

Over the past few years, there have been rapid advances 
Table 1 Comparison of localization duration and distance to the nodules among the 3 groups (211 nodes in total)

\begin{tabular}{lcc}
\hline & Duration of localization $(\mathrm{min})$ & Distance to the nodules $(\mathrm{cm})$ \\
\hline IOBSL group $(\mathrm{n}=66)$ & $5.106 \pm 1.069$ & $0.9357 \pm 0.354$ \\
ALL group $(\mathrm{n}=70)$ & $4.986 \pm 0.825$ & $0.9357 \pm 0.354^{\star}$ \\
IOBSL+ALL group $(\mathrm{n}=66)$ & $10.03 \pm 0.847^{\star \star *}$ & $0.8242 \pm 0.16^{\star \star}$ \\
\hline
\end{tabular}

${ }^{\star}, \mathrm{P}<0.05$, compared with the IOBSL group; ${ }^{\star \star}, \mathrm{P}<0.001$, compared with the IOBSL group; ${ }^{*}, \mathrm{P}<0.001$, compared with the ALL group.

IOBSL, intraoperative body surface localization; ALL, anatomical landmark localization.

in minimally invasive thoracic surgery, diagnostic imaging techniques, and understanding of small pulmonary nodules. The proportion of pulmonary nodule resection has gradually increased among VATS procedures as has the proportion of sublobectomy. Accordingly, localization has increasingly been required to enable precise resection. A key factor affecting the success of these surgeries is the ability to locate the pulmonary nodule easily, quickly, and precisely.

In recent years, the most important development of VATS is the development of positioning technology. Multiple techniques have been used to address the difficulty of intraoperative pulmonary nodules identification and to successfully perform VATS pulmonary wedge resection: (I) preoperative percutaneous placement of metal materials. such as hookwire and coils; (II) preoperative percutaneous injection of liquid materials, including methylene blue dye, contrast medium, and radionuclides; (III) various new techniques such as magnetic navigation and fluorescent staining; and (IV) palpation; (V) cone-beam CT (CBCT); (VI) intraoperative localization techniques, which are still under intensive research. Each these localization techniques have their unique strengths and weaknesses.

\section{Preoperative percutaneous placement of hookwire}

The double-barbed hookwire is a 20-gauge puncture needle that can directly pass through lung tissue to anchor the lesion. Initially developed for the localization of breast nodules, it has been widely applied for the localization of pulmonary nodules in China since 2009 (2). More specifically, after administration of local anesthesia, the cannula needle of the hookwire is positioned percutaneously into the lung parenchyma close to the targeted nodule. However, multiple CT scans need to be performed to ensure the precision of localization. This method is easy to implement and has a high success rate. It can effectively avoid conversion to thoracotomy, save surgical time, and increase the accuracy of pathological examination (3).
The cannula needle should be placed near the lesion, but penetration of the needle into the lesion should be avoided to maintain the integrity of the lesion and to avoid the implantation and dissemination of tumor cells. As an invasive technique, the hookwire method is associated with several complications including pneumothorax (7.5-49.1\%) (4), pain, hemothorax, and hookwire dislodgement $(4-22 \%)(5,6)$. To handle dislodgement, many strategies has been invented. The chest can be closed and redirected by CT. But this approach takes a long time. Prolonged excision is also an option. However, this will damage the patient's lung function and affect the prognosis. The remedial localization technique is also reported to be a good way for relocation (7). In addition, pulling and traction when moving the patient may aggravate lung injury and lead to severe chest pain and pleural reaction.

\section{Percutaneous CT-guided injection of methylene blue dye}

By virtue of its good visibility, methylene blue can help the operator to quickly and accurately locate lung nodules during the operation, avoiding excessive exposure of the patient to radiation. It is further characterized by its low cost, minimal equipment requirements for localization, easy operation, and low incidence of complications. However, due to the rapid diffusion rate of methylene blue, the surgery must be completed within a short period of time after localization. Moreover, methylene blue staining can be easily disturbed by the pigments on the lung tissue surface, especially in long-term smokers. It has also been reported that the pain after methylene blue injection is more severe, which may be because the pleura is irritated by the dye (8). Indocyanine green is also a safety method to localize the subpleural nodules. Indocyanine Green's positioning accuracy is comparable to previously reported microcoil positioning and other technologies (9). Currently, similar localization techniques also include the injection of silicone oil or medical glue $(10,11)$, which 
can also be used in combination with hookwire insertion. These combinations can overcome a variety of hook-wireassociated complications, such as pneumothorax, chest pain, and needle dislodgement and displacement, and obviate the too-rapid diffusion and extensive coloring in the lungs and chest cavity (which result in inaccurate localization) typical of methylene blue injection.

\section{Magnetic navigation and fuorescent staining}

New-generation magnetic navigation and fluorescence staining techniques have been increasingly applied in localization. For instance, electromagnetic navigation bronchoscope (ENB) is a new method that combines electromagnetic navigation, bronchoscopy, and other $3 \mathrm{D}$ reconstruction techniques. In this technique, an extracorporeal magnetic localization plate is used to guide the endobronchial probe for target lesion localization and biopsy. The high success rate of the ENB technique for intraoperative localization has been well demonstrated. Brown et al. (12), Awais et al. (13), Luo et al. (14), and Wang et al. (15) confirmed that ENB was valuable in the localization and resection of pulmonary nodules. ENB can be performed before surgery, with high accuracy and few complications (16); however, its application is limited by its high cost and other conditions.

\section{Palpation}

Palpation may detect pulmonary nodules not detected by preoperative imaging, and thoracotomy after palpation has no significant survival benefit compared with complete thoracoscopic resection of all visible nodules. Palpation is necessary when diagnostic imaging is used to define the extent and location of the nodule and to determine the surgical strategy to ensure complete removal of all nodules. One study showed that in 26 percent of patients, the number of lung lesions detected in preoperative CT scans correlates poorly with surgical findings, underestimating the number of metastases that survived, and thus the palpation is necessary in surgery. A study has reported that palpation failure during VATS is as high as $63 \%$ if the diameter of the nodule is less than $10 \mathrm{~mm}$ or if the pulmonary pleural is $>5 \mathrm{~mm}$ deeper from visceral pleura. The palpation seems inconvenient in VATS (17-19). As the incidence of pulmonary nodules, it is hard to localize nodules via palpation. Thus, other positioning methods with high accuracy are particularly important. Single-stage image- guided video-assisted thoracoscopic surgery is also feasible.

\section{CBCT}

CBCT has shown good results in the surgical anatomy of the lesion site and surrounding area, with a mean error of less than $2 \mathrm{~mm}$. The Hybrid Operation Room with CBCT provides perfect real-time imaging of patients. Positioning and excision can be performed in the same room. This method can effectively reduce the incidence of pneumothorax, metal marker dislocation and dye diffusion. Single-stage image-guided VATS for simultaneous localization and removal of small solitary pulmonary nodules is feasible $(20,21)$.

Each localization method has its advantages and disadvantages. Nodules smaller than $1.5 \mathrm{~cm}$ in diameter and less than $2 \mathrm{~cm}$ from the pleura are more difficult to reach during sublobectomy. For these lesions, a localization method that can precisely locate the nodules with reduced cost, radiation exposure, and pain is particularly important. In our center, there have been patients who refused surgery, as they were required to visit the radiology department for preoperative CT-guided invasive localization; there have also been cases in which the patient's blood pressure was abnormally high during the waiting period for surgery after preoperative localization, and the surgery had to be suspended. Thus, we have attempted to develop a new, simple, accurate, and noninvasive, intraoperative localization technique to compensate for the disadvantages of the preoperative methods.

For pulmonary nodules to be removed through sublobectomy, intraoperative localization can be performed by noninvasive or minimally invasive means in most cases if the visceral pleurae are shallow. IOBSL, as shown in one of our previous studies (22), can be performed using trocar needles. The IOBSL is proved to have a satisfied accuracy and low complications (23). In patients with a thick chest wall or in whom the lesions are blocked by the clavicle, IOBSL, ALL, or IOBSL+ALL may be applied.

The precision of IOBSL can be influenced by intraoperative body position and the degree preoperative CT breath-holding, and intraoperative status may change after lung inflation under anesthesia. We conducted the positioning under general anesthesia. The lung was inflated and kept in fixed size manually. Thus, breathing has little effect on orientation. As a result, IOBSL has a particularly large deviation in terms of distance. In contrast, ALL is based on human anatomical landmarks, which helps to 

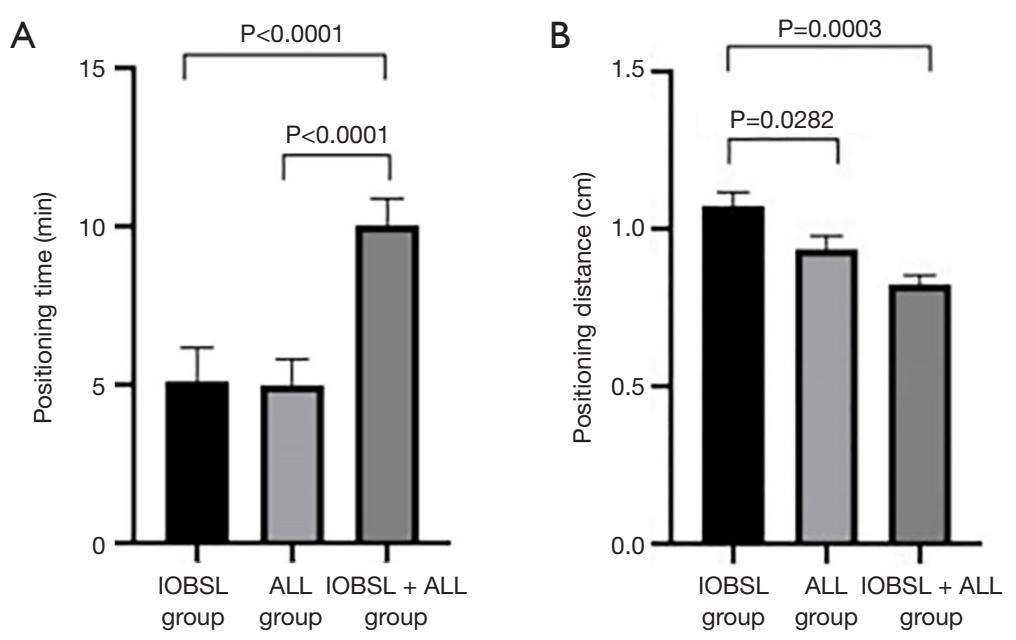

Figure 4 Comparison of localization duration and distance to the nodules among 3 groups. IOBSL, intraoperative body surface localization; ALL, anatomical landmark localization.

determine the approximate locations of the lung nodules. As surgeries rely on anatomical localization to determine the operating site, the accuracy of each operational step can be ensured. While ALL plays an important role in surgery, its value is obviously limited for small nodules. A collapsed lung during the surgery has quite a different anatomical location than the anatomical location of the normal lungs in the expanded state, and the density and hardness of the lesions are often difficult to distinguish from the adjacent lung tissues under such conditions. Therefore, the combination of IOBSL and ALL may further improve the accuracy of nodule localization. In our current study, we used different intraoperative localization methods to achieve better localization results: compared with the IOBSL group, the ALL group showed a shorter distance to the nodules $(\mathrm{P}=0.0282)$ but longer localization time $(\mathrm{P}<0.05)$, while the IOBSL+ALL group had higher localization precision than did the other groups $(\mathrm{P}=0.0003)$ but with longer duration $(\mathrm{P}<0.001)$ (Figure 4).

The advantages of intraoperative localization are described below: (I) real-time intraoperative localization rarely leads to pneumothorax, hemothorax, or other complications. (II) The use of a localization needle and electrocoagulation hook with electrocoagulation allows for operation in real time and for implantation and dissemination of tumor cells to be completely avoided. (III) When the lungs are inflated, they are in the same status as after deep inspiration and breath-holding during CT examination, which is conducive to accurate localization. (IV) The patients do not require transfer to the radiology department for localization and thus avoid long-duration radiation exposure. (V) The localization is performed after anesthesia in the operating room, avoiding psychological or physical trauma to the patient. (VI) The procedure is simple, safe, practical.

Some of our experiences in performing this technique are described below. (I) The preoperative measurement of the distance from the nodes to the pleura and chest wall should follow the principles of "vertical measurement" and "shortest distance". (II) During the puncture for localization, any injury to the bony thoracic structures should be avoided; meanwhile, the principle of "vertical needling" should be followed; that is, the angle between the localization needle and the parietal pleura should be as close as possible to $90^{\circ}$ to ensure that the result is consistent with the preoperative measurement. (III) After adequate anesthesia, the lungs are inflated and exhausted several times, and electrocoagulation should be used for localization under a natural expiration status to reduce deviation. (IV) After localization by electrocoagulation, one more stitch should be made at the localization point to facilitate the search for the lesion after excision. (V) When wedge resection is performed, cutting along the interlobar fissure or bronchi as much as possible can reduce small bronchial dissection, thus reducing postoperative scarring of lung tissue and promoting recovery of lung function.

An ideal localization method should have high accuracy, few complications, and a short procedure time; it should be easily mastered by beginners; it should be applicable to pulmonary nodules in all locations; and finally, it should be 
simple and have low technical requirements, avoiding the transfer of patients between the localization area and the operating room. IOBSL is very close to an ideal localization technique, but also has a few limitations. For instance, it has decreased accuracy for deeply situated pulmonary nodules with a distance of more than $2 \mathrm{~cm}$ from the visceral pleura. We will continue to investigate ways on how to further improve the accuracy of localization in our future studies.

\section{Acknowledgments}

Funding: Supported by Zhejiang Provincial Medical and Health Science and Technology Program (2019KY235).

\section{Footnote}

Reporting Checklist: The authors have completed the STROBE reporting checklist. Available at https://dx.doi. org/10.21037/tcr-21-1059

Data Sharing Statement: Available at https://dx.doi. org/10.21037/tcr-21-1059

Conflicts of Interest: All authors have completed the ICMJE uniform disclosure form (available at https://dx.doi. org/10.21037/tcr-21-1059). The authors have no conflicts of interest to declare.

Ethical Statement: The authors are accountable for all aspects of the work in ensuring that questions related to the accuracy or integrity of any part of the work are appropriately investigated and resolved. All procedures performed in this study involving human participants were in accordance with the Declaration of Helsinki (as revised in 2013). The study was approved by the ethics committee of Zhoushan Hospital with an approval number of [2018] ZHECA [163]. Individual consent for this retrospective analysis was waived.

Open Access Statement: This is an Open Access article distributed in accordance with the Creative Commons Attribution-NonCommercial-NoDerivs 4.0 International License (CC BY-NC-ND 4.0), which permits the noncommercial replication and distribution of the article with the strict proviso that no changes or edits are made and the original work is properly cited (including links to both the formal publication through the relevant DOI and the license). See: https://creativecommons.org/licenses/by-nc-nd/4.0/.

\section{References}

1. Wang L, Li WJ, Li YL, et al. Clinical value of intraoperative lung anatomical localization in totally thoracoscopic resection of pulmonary nodules. Zhejiang Medicine 2020;42:64-6.

2. Iguchi T, Hiraki T, Gobara H, et al. Transfissural route used for preoperative localization of small pulmonary lesions with a short hook wire and suture system. Cardiovasc Intervent Radiol 2015;38:222-6.

3. Xu C, Yuan Q, Wang Y, et al. Usefulness of virtual bronchoscopic navigation combined with endobronchial ultrasound guided transbronchial lung biopsy for solitary pulmonary nodules. Medicine (Baltimore) 2019;98:e14248.

4. Yoshida Y, Inoh S, Murakawa T, et al. Preoperative localization of small peripheral pulmonary nodules by percutaneous marking under computed tomography guidance. Interact Cardiovasc Thorac Surg 2011;13:25-8.

5. Pittet O, Christodoulou M, Pezzetta E, et al. Videoassisted thoracoscopic resection of a small pulmonary nodule after computed tomography-guided localization with a hook-wire system. Experience in 45 consecutive patients. World J Surg 2007;31:575-8.

6. Hänninen EL, Langrehr J, Raakow R, et al. Computed tomography-guided pulmonary nodule localization before thoracoscopic resection. Acta Radiol 2004;45:284-8.

7. Wang G, Zhu X, Zhang F, et al. Applied research of a fourdimensional CT localization technique in radiotherapy and treatment planning for early lung cancer. Transl Cancer Res 2020;9:7227-35.

8. Kleedehn M, Kim DH, Lee FT, et al. Preoperative Pulmonary Nodule Localization: A Comparison of Methylene Blue and Hookwire Techniques. AJR Am J Roentgenol 2016;207:1334-9.

9. Xu X, Yao Y, Shen Y, et al. Clinical Analysis of Percutaneous Computed Tomography-Guided Hook Wire Localization of 168 Small Pulmonary Nodules. Ann Thorac Surg 2015;100:1861-7.

10. Jin KN, Lee KW, Kim TJ, et al. Computed tomography guided percutaneous injection of a mixture of lipiodol and methylene blue in rabbit lungs: evaluation of localization ability for video-assisted thoracoscopic surgery. J Korean Med Sci 2014;29:129-36.

11. Shentu Y, Zhang L, Gu H, et al. A new technique combining virtual simulation and methylene blue staining for the localization of small peripheral pulmonary lesions. BMC Cancer 2014;14:79.

12. Brown J, Lee TJ, Joiner T, et al. Using Electromagnetic 
Navigation Bronchoscopy and Dye Injection to Aid in

Video-Assisted Lung Resection. Am Surg 2016;82:1052-4.

13. Awais O, Reidy MR, Mehta K, et al. Electromagnetic Navigation Bronchoscopy-Guided Dye Marking for Thoracoscopic Resection of Pulmonary Nodules. Ann Thorac Surg 2016;102:223-9.

14. Luo K, Lin Y, Lin X, et al. Localization of peripheral pulmonary lesions to aid surgical resection: a novel approach for electromagnetic navigation bronchoscopic dye marking. Eur J Cardiothorac Surg 2017;52:516-21.

15. Wang G, Lin Y, Luo K, et al. Feasibility of injecting Fluorescent Agent under the Guidance of Electromagnetic Navigation Bronchoscopy in Pulmonary Nodule Resection. Zhongguo Fei Ai Za Zhi 2020;23:503-8.

16. Sun J, Mao X, Xie F, et al. Electromagnetic navigation bronchoscopy guided injection of methylene blue combined with hookwire for preoperative localization of small pulmonary lesions in thoracoscopic surgery. J Thorac Dis 2015;7:E652-6.

17. Kayton ML, Huvos AG, Casher J, et al. Computed tomographic scan of the chest underestimates the number of metastatic lesions in osteosarcoma. J Pediatr Surg 2006;41:200-6; discussion 200-6.

18. Lautz TB, Krailo MD, Han R, et al. Current surgical management of children with osteosarcoma and pulmonary metastatic disease: A survey of the American Pediatric Surgical Association. J Pediatr Surg 2021;56:282-5.

19. Suzuki K, Nagai K, Yoshida J, et al. Video-assisted thoracoscopic surgery for small indeterminate pulmonary nodules: indications for preoperative marking. Chest 1999; 115:563-8.

20. Zhao ZR, Lau RWH, Yu PSY, et al. Devising the guidelines: the techniques of pulmonary nodule localization in uniportal video-assisted thoracic surgeryhybrid operating room in the future. J Thorac Dis 2019;11:S2073-8.

21. Chen J, Pan X, Gu C, et al. The feasibility of navigation bronchoscopy-guided pulmonary microcoil localization of small pulmonary nodules prior to thoracoscopic surgery. Transl Lung Cancer Res 2020;9:2380-90.

22. Zhang BJ, Zhang YK, Le HB, et al. A research on the application of body surface localization in the treatment of pulmonary nodules during thoracic surgery. China Oncology 2017;27:976-9.

23. Su TH, Fan YF, Jin L, et al. CT-guided localization of small pulmonary nodules using adjacent microcoil implantation prior to video-assisted thoracoscopic surgical resection. Eur Radiol 2015;25:2627-33.

(English Language Editor: J. Gray)
Cite this article as: Zhang B, Zhang Y, Le H, Li W, Chen C, Fang R, Pan X. Intraoperative localization in minimally invasive surgery for small pulmonary nodules: a retrospective study. Transl Cancer Res 2021;10(7):3470-3478. doi: 10.21037/tcr-21-1059 